\title{
Periazetabuläre Frakturen bei Hüftprothesen
}

\author{
Marius Johann Baptist Keel, Johannes Dominik Bastian
}

\section{Zusammenfassung}

Periazetabuläre Frakturen bei Hüftprothesen nehmen aufgrund der Überalterung und der zunehmenden Aktivität alter Menschen zu. Die periprothetischen Azetabulumfrakturen werden anhand der Einteilung von Letournel klassifiziert. Wenn beide Azetabulumpfeiler bei Hüftprothese betroffen sind, wird auch von einer Beckendiskontinuität gesprochen. Durch eine laterale Kompression können auch periazetabuläre Schambeinastfrakturen und/ oder transiliakale Frakturen auftreten. Für die Therapieentscheidung (konservativ, alleinige Osteosynthese, Revisionshüfttotalprothese mit oder ohne zusätzliche Osteosynthese des Vorderund/oder Hinterpfeilers) und die Zugangswahl bei operativer Versorgung werden patientenspezifische (Alter, Morbidität, Osteoporose, Aktivitätslevel des Patienten), frakturspezifische (Frakturtyp, Dislokationsausmaß, Impression des Doms oder der Hinterwand) und auch prothesenspezifische Faktoren (Art der implantierten Prothese [Hemiprothese vs. Totalprothese], Pfannenstabilität, Zeichen eines Prothesenabriebs, Ausmaß und Lokalisation einer azetabulären Lyse, Stabilität und Lysezeichen des Prothesenschafts) berücksichtigt. Bei akuten Beckendiskontinuitäten werden neben einer Osteosynthese des dorsalen Pfeilers zunehmend eine schnell ossär integrierbare Pfanne (Tantalum [„Trabecular Metal“: TM]) mit oder ohne Augment und/oder Allograft und allenfalls in einer sog. „Cup-Cage“-Technik (TMPfanne mit einem abstützenden Revisionsring [Burch-Schneider-Ring] analog zur Therapie von chronischen Beckendiskontinuitäten empfohlen. Bei großen Lysezonen und starken Dislokationen des vorderen Pfeilers und der quadrilateralen Fläche können intrapelvine Zugänge (modifizierter Stoppaoder Pararectus-Zugang nach Keel) zur zusätzlichen Zuggurtungsosteosynthese des vorderen Pfeilers und Abstützung der quadrilateralen Fläche gewählt werden.

\section{Periacetabular Fractures in Patients} with Hip Arthroplasties

The incidence of periacetabular fractures in patients with hip arthroplasties is increasing due to the ageing population and the high activity of the elderly. The periprosthetic acetabular fractures are classified according to the classification of Letournel. A periprosthetic acetabular fracture with an involvement of both columns is also called a pelvic discontinuity. Lateral compression injuries can also occur as periacetabular pubic fractures and/or transiliac frac- tures. Patient-related (age, morbidity, osteoporosis, activity level of the patient), fracture-related (type of fracture, grade of dislocation, impaction of the dome or the posterior wall) as well as prosthesis-related factors (type of prosthesis [hemi-arthroplasty or total arthroplasty], stability of the cup, wear of the prosthesis, grade and localisation of the acetabular lysis, stability or lysis of the prosthesis stem) are considered for the decision of the definitive treatment (conservative, isolated osteosynthesis, revision total hip arthroplasty with or without additional osteosynthesis of the anterior and/or posterior columns) and the choice of the surgical approach. For acute pelvic discontinuities an osteosynthesis of the posterior column and a highly osteointegrative cup (tantalum [trabecular metal: TM]) with or without augmentation and/or allograft and in some complex cases in a cup-cage technique (TM-cup with a reconstructive cage [Burch-Schneider cage]) are recommended according to the therapeutic concept for chronic pelvic discontinuties. In cases with severe acetabular lysis and displacements of the anterior column and quadrilateral plate an additional internal fixation of them through an intrapelvic approach (modified Stoppa approach or Pararectus approach [Keel]) is becoming more popular.

\section{Einleitung}

Aufgrund der Überalterung und der zunehmenden Aktivität alter Menschen werden periazetabuläre Frakturen bei Hüftprothesen vermehrt beobachtet [6, 11]. Neben eigentlichen periprotheti-

OP-JOURNAL 2015; 31: 15-21

(c) Georg Thieme Verlag KG Stuttgart · New York DOI http://dx.doi.org/10.1055/s-0035-1545871 schen Azetabulumfrakturen können auch periazetabuläre Schambeinastfrakturen oder seltener transiliakale Frakturen auftreten. Als Ursache steht der seitliche Sturz auf den Trochanter major (Sturz als Fußgänger, Velofahrer oder bei sportlichen Aktivitäten [z.B. Skifahren]) beim geriatrischen Patienten mit oder ohne Osteoporose im Vordergrund. Seltener entstehen diese Frakturen bei Verkehrsunfällen, bei einem Sturz aus großer Höhe (Suizid, Wandern) oder iatrogen beim Einschlagen einer Pressfit-Pfanne, vor allem nach zu tiefem Auffräsen des Azetabulums [13].

Neben der Osteoporose ist ein vorbestehender aseptischer oder septischer Knochenverlust um die Prothesenpfanne ein großer Risikofaktor für eine periazetabuläre Fraktur.

Die Pfanne kann schon vor dem Ereignis locker sein oder noch stabil im Os ischi- 
um oder Os pubis integriert sein. Die Lysezonen können anhand der AAOS- oder der Paprosky-Klassifikation beschrieben werden (AAOS: American Academy of Orthopaedic Surgeons) $[5,11,12]$. Ob der Knochendefekt im Vorderpfeiler, Hinterpfeiler mit evtl. auch fehlender Hinterwand und/oder im Dombereich liegt, hat einen Einfluss auf die Art der Beckenstabilisierung und der azetabulären Rekonstruktion [13]. Ebenfalls bedeutend für das Therapiekonzept ist, ob die periazetabuläre Farktur um eine Hemiprothese oder eine Totalprothese entstanden ist.

Grundsätzlich können die periprothetischen Azetabulumfrakturen anhand der Klassifikation von Letournel eingeteilt werden [10]. Beim seitlichen Sturz treten beim alten Menschen mit Hüftprothesen wie bei nativem Hüftgelenk isolierte Vorderwand- oder Vorderpfeilerfrakturen oder häufiger Vorderpfeilerfrakturen mit posteriorer Hemiquerfraktur, Querfrakturen, T-förmige Frakturen oder Zweipfeilerfrakturen häufig mit Ausbruch des Azetabulumbodens (quadrilaterale Fläche) auf $[2,6,8,9]$. Außer bei den isolierten Vorderwand- oder Vorderpfeilerfrakturen wird von einer

akuten Beckendiskontinuität gesprochen, falls die Fraktur anamnestisch innerhalb der letzten 12 Wochen entstanden ist [14]. Dabei sind beide Azetabulumpfeiler unterbrochen und der superiore Beckenanteil ist vom distalen vollständig getrennt.

Bei großen Lysezonen tritt die Dislokation nach zentral oder kranial häufig kontinuierlich über mehrere Wochen auf, weshalb dann von einer chronischen (>12 Wochen) Beckendiskontinuität gesprochen wird. In den nachfolgenden Ausführungen wird nur auf die Therapie der periprothetischen Azetabulumfrakturen eingegangen, da die Versorgung von periazetabulären Schambeinastoder transiliakalen Frakturen bei Hüftprothesen gemäß den Prinzipien der konservativen oder operativen Therapie von Beckenringfrakturen durchgeführt wird.

\section{Diagnostik}

Klinisch präsentiert sich eine periprothetische Azetabulumfraktur durch eine schmerzhafte Einschränkung der Hüftbeweglichkeit und eine schmerzhafte oder unmögliche Belastbarkeit des Beines. Auch Fernschmerzen im Oberschen- kel können Ausdruck einer periprothetischen Azetabulumfraktur sein. Selten wird die Diagnose einer periprothetischen Fraktur im Rahmen einer Luxation gestellt. Bei einer dorsalen Luxation besteht eine fixierte, federnde Fehlstellung des Beines in Innenrotation. Bei wachem Patienten muss bei dorsaler Luxation immer eine mögliche Läsion des N. ischiadicus geprüft werden (Sensibilitätsverlust am Fußrücken und Zehen- und Fußheberschwäche).

Bei älteren Patienten werden jedoch häufiger aufgrund des lateralen Kompressionsmechanismus Frakturen mit medialisierter Hüftpfanne beobachtet,

was ebenfalls zu einer schmerzhaften Einschränkung der Hüftbeweglichkeit führt. Bei einer eigentlichen zentralen Luxation der Hüftpfanne kann es durch das extraartikuläre Impingement des Trochanter minor und major zur zusätzlichen dorsalen Luxation des Schaftes kommen, evtl. auch begleitet von einem Trochanterabriss. Eine stark medialisierte quadrilaterale Fläche kann nicht selten von einer hämodynamisch relevanten Blutung begleitet sein.

Zur radiologischen Diagnostik gehören eine Beckenübersicht, eine symphysenzentrierte Beckenaufnahme und eine axiale Hüftgelenksaufnahme. Neben den klassischen Leitlinien zur Klassifikation der Azetabulumfraktur müssen auch Zeichen einer Lockerung der Hüftgelenkspfanne oder des Prothesenschafts gesucht werden. Eine medialisierte Prothesenpfanne, eine dislozierte quadrilaterale Fläche oder eine dislozierte Tränenfigur sind Zeichen einer gelockerten Pfanne. Eine veränderte Position einer Pfanne lässt sich gut anhand eines Vergleiches mit früheren Röntgenbildern eruieren.

Die 6 Leitlinien (Linea iliopectinea [Vorderpfeiler], Linea ilioischiadica [Hinterpfeiler], Vorderwand, Hinterwand, Foramen obturatum und Ala ossis ilii) in der Beckenübersicht erlauben im Ausschlussverfahren in über $90 \%$ die genaue Klassifizierung der Azetabulumfraktur gemäß Letournel mit den 10 verschiedenen Frakturtypen [10].

Falls sowohl die Linea iliopectinea als auch die Linea ilioischiadica unterbrochen sind, können alle isolierten Pfeileroder Wandfrakturen und deren auf einen Pfeiler isolierte Kombinationsfrakturen, wie Hinterpfeiler- mit Hinterwandfrak- tur, ausgeschlossen werden und nur noch eine Zweipfeilerfraktur, eine Vorderpfeilerfraktur mit posteriorer Hemiquerfraktur, eine T-förmige Fraktur oder eine Querfraktur mit oder ohne Hinterwandfraktur kommen infrage. Bei diesen Frakturformen spricht man von einer sog. Beckendiskontinuität [11,14]. Die zusätzliche Aufnahme mit Anhebung der nicht betroffenen Beckenhälfte um $45^{\circ}$ (Ala-Aufnahme) vereinfacht die Beurteilung einer möglichen Fraktur in der Beckenschaufel, eine Unterbrechung im vorderen Pfannenrand oder im hinteren Pfeiler (Linea ilioischiadica). Eine Anhebung der betroffenen Seite um $45^{\circ}$ (Obturator-Aufnahme) lässt eine einfachere Beurteilung des Foramen obturatum, des hinteren Pfannenrands oder des Vorderpfeilers (Linea iliopectinea) zu. Das Auftreten des Sporenzeichens („Spursign“) in der Obturator-Aufnahme ist pathognomonisch für eine Zweipfeilerazetabulumfraktur mit vollständiger Separation des Azetabulums und somit auch der Hüftprothese vom hinteren Beckenring [10]. In den Röntgenuntersuchungen sollte zudem bei schon länger implantierten Hüftprothesen nach Zeichen eines Prothesenabriebs wie Lysezonen im azetabulären Knochen oder ein exzentrisch liegender Prothesenkopf im Polyethylen-Inlay gesucht werden.

Die Klassifikation einer periprothetischen Azetabulumfraktur und deren Beurteilung bez. Therapieentscheidung in der Beckenübersicht mit oder ohne Alaund Obturator-Aufnahmen ist bei einer vorbestehenden Hüftprothese jedoch wesentlich schwieriger als bei einem nativen Hüftgelenk.

Deshalb sollte immer eine Computertomografie im Rahmen der Akutdiagnostik bei schmerzhafter Hüftprothese nach Sturz durchgeführt werden.

Bei den axialen Schnittbildern der Computertomografie entsprechen eine vertikale Frakturlinie einer Querfraktur, eine horizontale Linie ventral einer Fraktur im vorderen Pfeiler und eine solche dorsal einer Fraktur im hinteren Pfeiler. Neben den axialen Bildern helfen sagittale und koronare Rekonstruktionen bei der Beurteilung des Dislokationsausmaßes und der Komplexität der Fraktur (mehrfragmentär). Der Nachweis von intraartikulären Fragmenten, einer Domimpaktion oder einer dorsalen marginalen Impaktion ist allenfalls bei liegender Hemiprothese für die Therapieentscheidung (Osteosynthese vs. Osteosynthese 

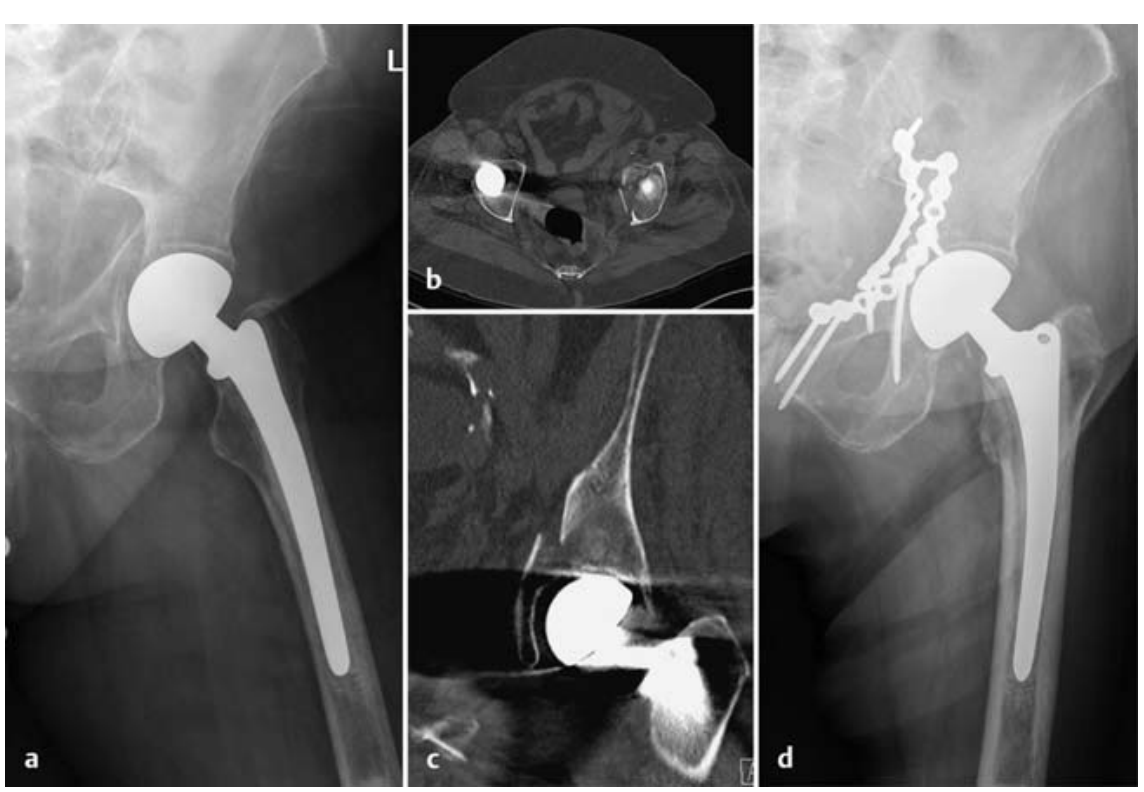

Abb. 1 a bis d Eine 85-jährige Patientin zog sich bei einem seitlichen Sturz eine periprothetische Azetabulumfraktur (Vorderpfeiler- mit posteriorer Hemiquerfraktur) bei liegender monopolarer Hemiprothese zu: a Hüfte a.-p. und b axiale Computertomografie (CT) resp. c koronares CTSchnittbild. d Vollständige Konsolidation der Fraktur und stabile Hemiprothese bei erhaltenem Azetabulum 1 Jahr postoperativ nach offener Reposition und Osteosynthese über einen intrapelvinen Zugang (Pararectus) bei beschwerdefreier Patientin.

und Revisionsprothese) von Bedeutung. Zudem sind diese zusätzlichen Kriterien bei liegender Hüftprothese aufgrund von Metallartefakten kaum sichtbar. Bedeutender bei periprothetischen Azetabulumfrakturen ist in der Computertomografie die Beurteilung der Stabilität der Hüftpfanne (Lockerung von Schrauben bei Rekonstruktionspfannen oder Spaltbildungen bei Pressfit-Pfannen) zum hinteren oder vorderen Azetabulumpfeiler. Daneben sollte das Ausmaß von Lysezonen bei Verdacht auf Lockerungen der Hüftpfanne im vorderen und hinteren Pfeiler oder im Dom beschrieben werden und gemäß der AAOS-Klassifikation oder nach Paprosky graduiert werden $[5,12]$. Zudem müssen neben Lysezonen um die Pfanne auch solche im Schaftbereich gesucht werden. Durch die ergänzende Durchführung eines SPECT (single photon emission tomography/computed tomography)/CT können Zusatzinformationen bez. der Stellung der Prothesenkomponenten, Ausdehnung von Osteolysen, Verlauf von periprothetischen Frakturen und der Weichteilsituation (Muskelatrophie, Erguss) gewonnen oder ergänzt werden. Diese Technik erlaubt es, gezielte szintigrafische und CT-Schichtbilder in allen Raumebenen zu entwickeln [15].

\section{Behandlungskonzepte bei Hemiprothese}

Hemiprothesen, in monopolarer oder bipolarer Form, werden aufgrund von dislozierten Schenkelhalsfrakturen bei sehr betagten Patienten oder solchen mit schweren Vorerkrankungen implantiert, wenn der azetabuläre Knorpel noch intakt ist und der Patient nicht vorbestehend über Hüftschmerzen klagte.

Falls nun bei einem erneuten Sturz eine periprothetische Azetabulumfraktur bei liegender Hemiprothese entsteht, muss grundsätzlich entschieden werden, ob die Hemiprothese belassen werden kann oder ein Wechsel zu einer Totalprothese notwendig ist.

Als Entscheidungsfaktoren dienen, neben dem Dislokationsausmaß der Azetabulumfraktur oder dem Risiko einer sekundären Dislokation bei un- oder wenig dislozierter Azetabulumfraktur, die Knochenqualität (Osteoporose), das Aktivitätslevel des Patienten unabhängig vom primären Alter und die Vorerkrankungen des Patienten hinsichtlich einer möglichen operativen Belastung und der Lebenserwartung. Daneben sollte in der Therapieentscheidung auch ein Verfahren gewählt werden, das möglichst keine sekundären Eingriffe notwendig macht und eine frühzeitige Vollbelastung erlaubt. Nicht zu vergessen ist, dass mit der steigenden Häufigkeit von Eingriffen bei vorbestehender Hüftprothese das Risiko für eine Infektion mit komplikationsreichem Outcome (Prothesenluxation, Immobilität bei GirdlestoneSituation) steigt. Als Therapieoptionen sind neben der konservativen Therapie mit Entlastung (Teilbelastung oder Rollstuhlmobilität) bei nicht dislozierten Frakturen eine offene Reposition und stabile Osteosynthese ohne Wechsel auf eine Totalprothese bei wenig dislozierten Frakturen oder bei Patienten mit hoher Morbidität (kaum mehr gehfähige Patienten, kurze Lebenserwartung) möglich (Abb. 1). Bei stark dislozierten Frakturen und solchen mit deutlicher Domimpression oder dorsaler Impaktion resp. Luxationsfrakturen sollte ein Wechsel auf eine Hüfttotalprothese durchgeführt werden, wobei je nach Frakturmuster eine begleitende Osteosynthese im vorderen oder hinteren Pfeiler notwendig ist (Abb. 2).

Durch die Einführung von intrapelvinen Zugängen (modifizierter Stoppa-Zugang, Pararectus-Zugang nach Keel) können die typischen Frakturen mit medialisierter quadrilateraler Fläche einfacher versorgt (kleinerer Zugang) und stabiler fixiert (innere Abstützplatte) werden als durch den ilioinguinalen Zugang $[2,4,8,9]$.

Je nach Ausmaß des Knochendefekts muss zusätzlich Allograft und eine Rekonstruktionspfanne verwendet werden. Als Alternative mit besseren Langzeitresultaten, vor allem bei älteren Patienten und bei sehr großen Defekten, können Implantate (Pfannen, Augments) eingesetzt werden, die ein schnelles Einwachsen des Knochens erlauben wie „Trabecular Metal“ (TM, Tantalum; Abb. 2) $[1,3,11,13,14]$. Bei intraoperativer Feststellung einer Luxationstendenz mit Ausschöpfung der Längen- und Offsetkorrektur, vermehrter Antetorsion des Schaftes, der Inklination und Anteversion der Pfanne sowie der Resektion von überstehendem Knochen am Trochanter major oder azetabulär zur Behebung eines extraartikulären Impingements kann zusätzlich eine „Dual-mobility“-Komponente als Luxationsprophylaxe einzementiert werden [7].

\section{Behandlungskonzepte bei Totalprothese}

Bei un- oder wenig dislozierten periprothetischen Azetabulumfrakturen bei Hüfttotalprothesen kann ebenfalls eine 


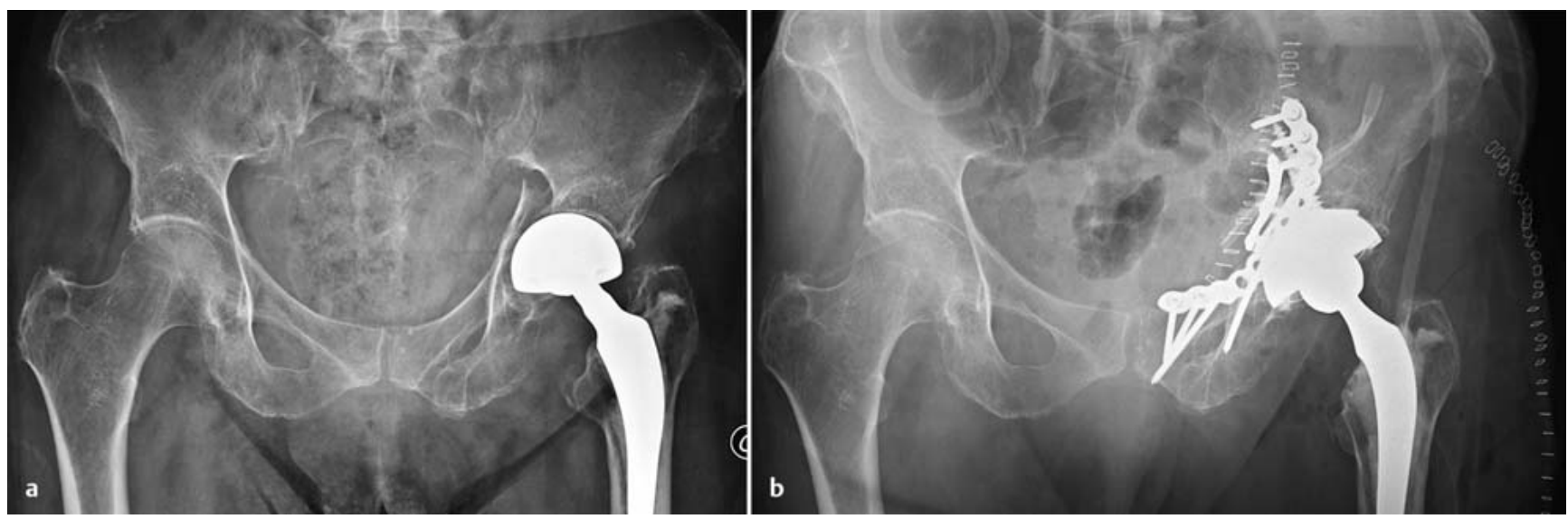

Abb. $\mathbf{2}$ a und b Eine 89-jährige Patientin mit einer zementierten Hemiprothese weist eine periprothetische Azetabulumquerfraktur mit medialisierter quadrilateraler Fläche nach einem Sturz auf (a Beckenübersicht). Bei noch hoher Alltagsaktivität wurde in Rückenlage eine offene Reposition und Plattenosteoynthese des vorderen Pfeilers mit Abstützung der quadrilateralen Fläche über einen Pararectus-Zugang durchgeführt. In der gleichen Operation wurde in Seitenlage über den vorbestehenden dorsalen Zugang eine TM-Pfanne (TM: „Trabecular Metal“) und ein zementierter Schaft mit mehr Antetorsion bei dorsaler Luxationstendenz implantiert (b Beckenübersicht postoperativ).
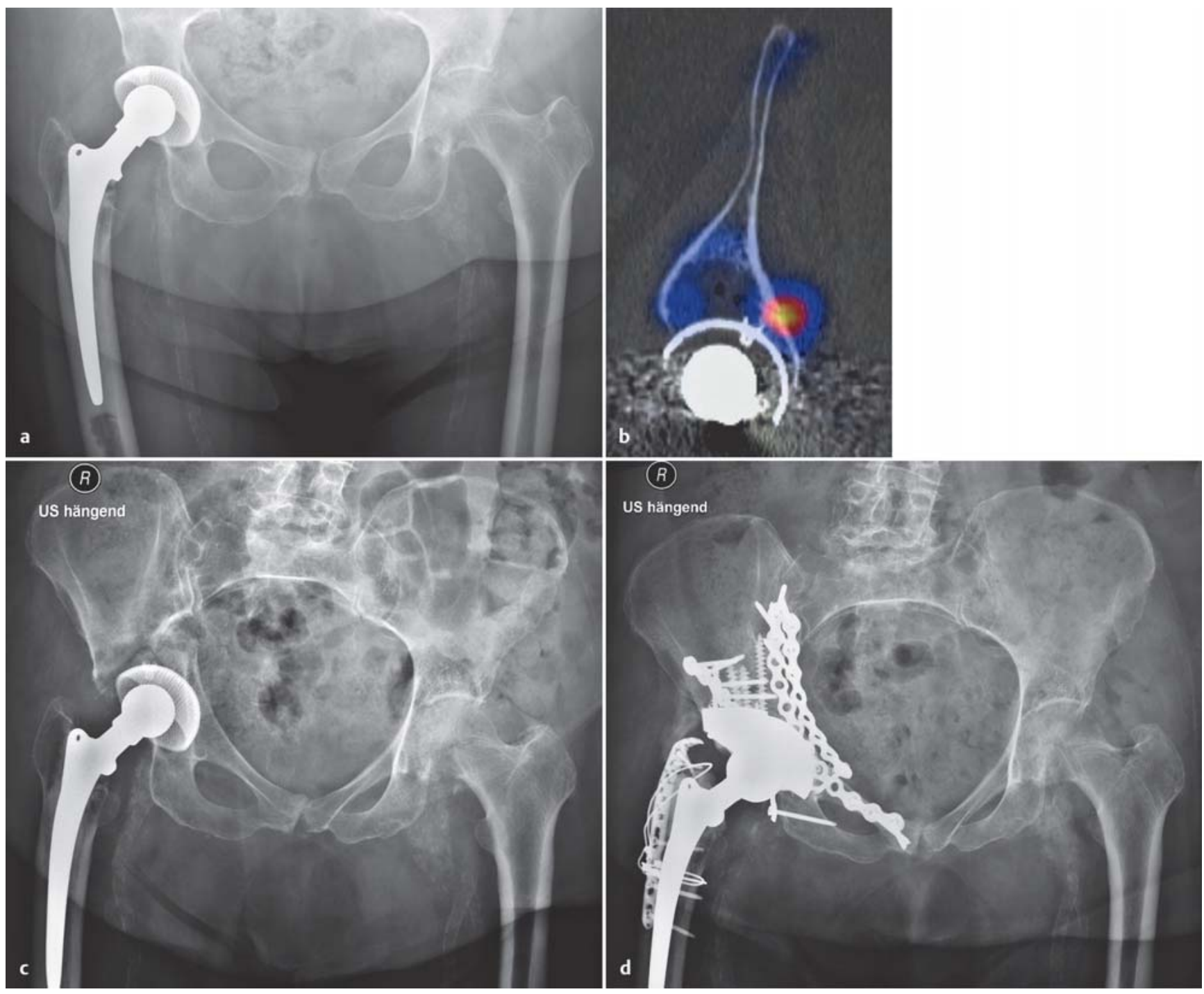

Abb. 3 a bis d Bei einer 85-jährigen Patientin mit Hüftschmerzen zeigt sich eine Lyse im Dombereich bei liegender Hüfttotalprothese (a). In einer SPECT/CT-Untersuchung zeigt sich eine Aktivitätszone als Ausdruck einer periprothetischen Azetabulumquerfraktur (b sagittale SPECT/CT). Nach 2 Wochen konservativer Therapie entwickelte sich eine deutliche Dislokation der Querfraktur bei großem Knochendefekt (c). Beckenübersicht 4 Monate postoperativ nach aufwendiger Rekonstruktion mit intrapelvinen Plattenosteosynthesen über einen Pararectus-Zugang und lateralen Zugang mit Osteosynthese des dorsalen Pfeilers, azetabulärem Aufbau (TM-Augment und Allograft) und TM-Pfanne sowie Trochanterfixation eines Trochanterabrisses (d). 

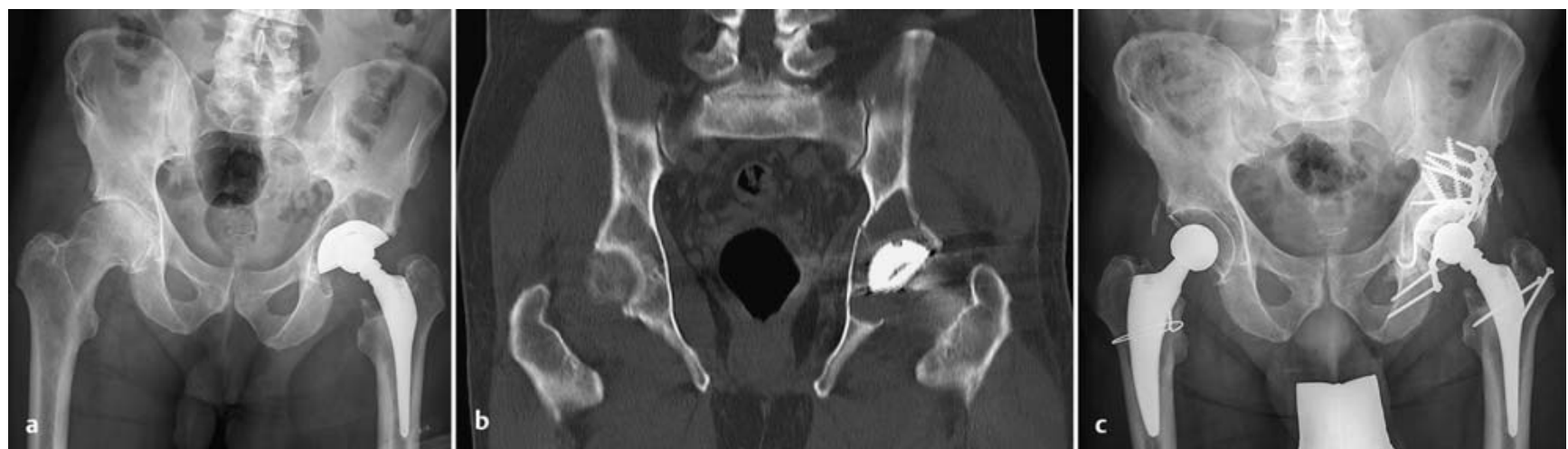

Abb. 4 a bis c Bei einem 67-jährigen Patienten entstand bei einem Bagatelltrauma bei vorbestehender großer azetabulärer Lyse im Hinterpfeiler und im Dom eine wenig dislozierte periprothetische Azetabulumquerfraktur (a Beckenübersicht und b koronarer CT-Schnitt). Beckenübersicht 2,5 Jahre nach Plattenosteosynthese des Hinterpfeilers und Auffüllen der Lyse mit Allograft sowie Implantation einer Rekonstruktionspfanne (Hakendachschale mit Lasche) links und 1 Jahr nach Implantation einer anterioren minimalinvasiven Hüfttotalprothese rechts bei Koxarthrose (c).
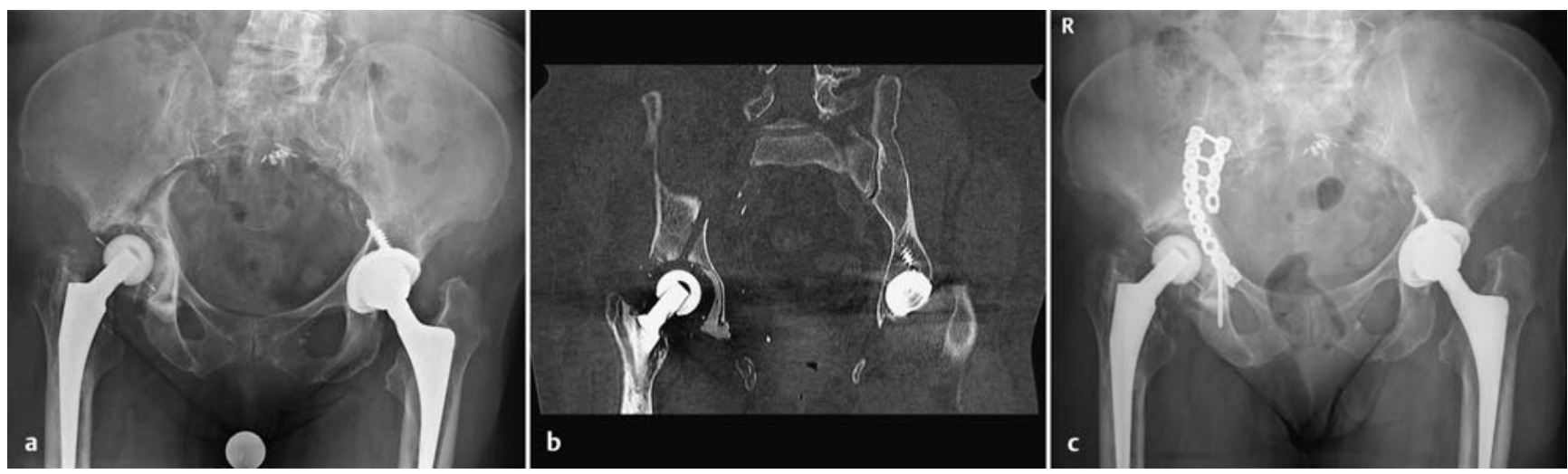

Abb. 5 a bis c Eine 69-jährige Patientin mit einer zementierten Hüfttotalprothesenpfanne zog sich bei einem Sturz eine dislozierte periprothetische Azetabulum-T-Fraktur mit jedoch stabiler Pfanne zu (a Beckenübersicht und b koronarer CT-Schnitt). Aufgrund der Morbidität der Patientin und der stabilen Pfannenposition wurde über einen Pararectus-Zugang eine offene Reposition und Plattenosteosynthese durchgeführt (c Beckenübersicht 6 Monate postoperativ).
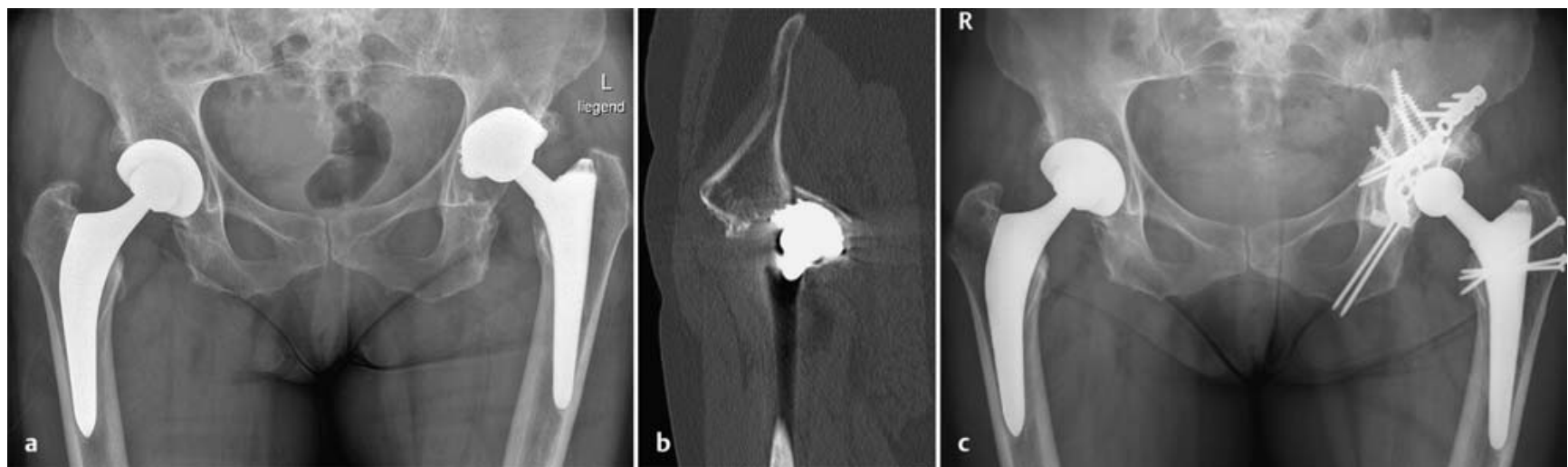

Abb. 6 a bis c Eine 78-jährige Patientin zog sich bei einem Sturz eine periprothetische mehrfragmentäre Azetabulumhinterwandfraktur mit dorsaler Luxation der Hüfttotalprothesenpfanne zu (a Beckenübersicht und b koronarer CT-Schnitt). Neben einer Implantation einer Rekonstruktionspfanne (Hakendachschale) wurde die Hinterwand rekonstruiert (c Beckenübersicht 1 Jahr postoperativ).

konservative Therapie mit Teilbelastung während 6-8 Wochen und radiologischen Verlaufskontrollen durchgeführt werden. Entscheidend ist die Stabilität der Prothesenpfanne.
Falls eine große Lysezone bei auch wenig dislozierter Fraktur periazetabulär vorhanden ist, besteht ein großes Risiko für eine sekundäre Dislokation (Abb. 3).

Diese Frakturen sollten primär mittels Osteosynthese des Hinterpfeilers, je nach Dislokationsausmaß auch des Vorderpfeilers, stabilisiert werden, der Knochendefekt mit strukturellem Allograft oder „Impaction grafting“ und/oder mit einem TM-Augment aufgefüllt werden („Down-grading“ des Defekts) und eine Revisionspfanne (Rekonstruktionsring 

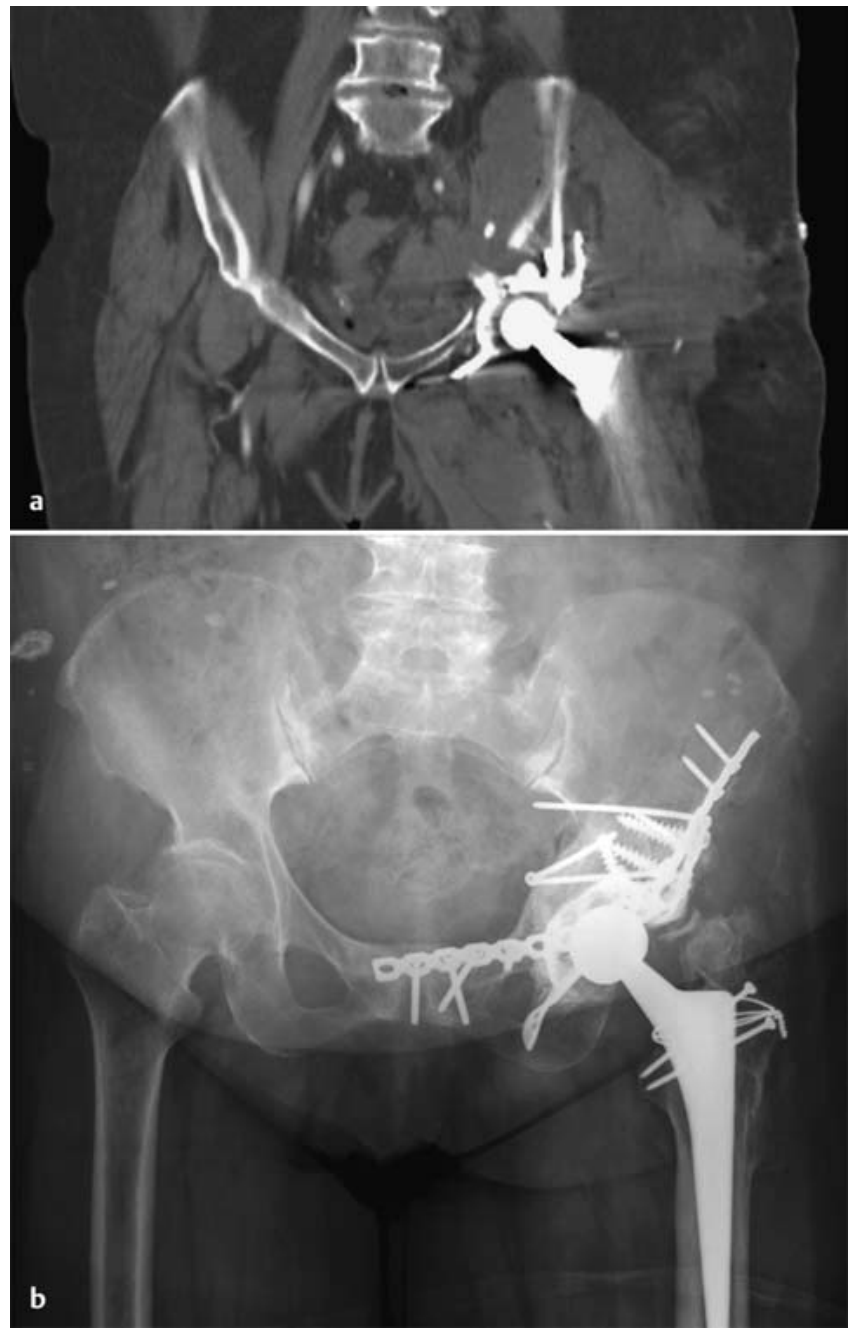

Abb. $7 \mathbf{a}$ und $\mathbf{b}$

Bei einer 73-jährigen Patientin mit primärer Koxarthrose entstand intraoperativ beim Setzen der Pressfit-Pfanne eine Azetabulumquerfraktur. Aufgrund der Fehlplatzierung eines Burch-Schneider-Ringes und einer deutlichen Blutung wurde die Patientin zur Revision in ein Zentrumsspital verlegt (a koronarer CT-Schnitt). b Beckenübersicht 4 Jahre nach Überbrückungsplatte durch einen StoppaZugang und lateralen Zugang mit Trochanterfliposteotomie, azetabulärem Aufbau mit strukturellem Allograft und Einsetzen eines Burch-Schneider-Ringes. und/oder TM-Pfanne) implantiert werden (Abb.4) [11,13]. Bei stark dislozierten Frakturen mit großem Knochendefekt ist der operative Aufwand jedoch häufig deutlich größer und entspricht der Herausforderung einer chronischen Beckendiskontinuität. Neben der Stabilisierung des Hinterpfeilers sollte zur Erhöhung der Beckenstabilität bei stark disloziertem Vorderpfeiler auch dieser mittels Plattenosteosynthese versorgt werden, damit der azetabuläre Aufbau mit Allograft und/oder Einsetzen von Augments sowie die Größe einer Revisionspfanne oder einer TM-Pfanne mit oder ohne zusätzlichen Rekonstruktionsring („Cup-Cage“-Technik) möglichst limitiert respektive klein gehalten werden kann (Abb. 3). Es wurde gezeigt, dass bei Patienten mit aseptischen Lockerungen und ausgedehnten Knochendefekten oder bei Patienten mit einer chronischen Beckendiskontinuität eine signifikant höhere Lockerungsrate nach Revisionsringen als nach TM-Pfannen zu beobachten ist $[1,3,11,13,14]$.
Auch wenn nach TM-Implantation erst ein kurzer Beobachtungszeitraum von weniger als 5 Jahren vorhanden ist, scheint das TM eine erhöhte Biokompatibilität mit verstärktem Einwachsen des Knochens und besserer Fixation aufzuweisen.

Bei periprothetischen Frakturen, bei denen die Prothesenpfanne noch stabil mit über $50 \%$ im Vorder- oder Hinterpfeiler integriert ist, kann eine reine Osteosynthese des Vorder- oder Hinterpfeilers ausreichen (Abb.5). Dies ist vor allem eine Option bei polymorbiden alten Patienten mit einem tiefen Aktivitätslevel. Periprothetische Frakturen mit dorsaler Subluxation oder Luxation der Pfanne und begleitender dorsaler Pfannenrandfraktur sind deutlich seltener als Frakturen mit medialisierter Pfanne. Die dorsale Pfannenrandfraktur muss über einen dorsalen Zugang stabilisiert werden und eine Revisionspfanne implantiert werden (Abb. 6). Bei osteoporotischem Knochen kann die Hinterwand auch durch ein TM-Augment ersetzt werden. Solche Frakturen können auch intraoperativ während der Implantation einer Pressfit-Pfanne bei primärer Koxarthrose auftreten. Ebenfalls kann intraoperativ eine echte Beckendiskontinuität auftreten, die adäquat wie oben beschrieben mittels Osteosynthese und Implantation eines Revisionsrings oder einer TMPfanne versorgt wird (Abb. 7). Eine seltene Herausforderung ist ein sehr großer ossärer Defekt azetabulär bei einer Beckendiskontinuität aufgrund eines zentral luxierten Zementspacers im Rahmen einer Therapie bei infizierter Hüftprothese. In einer solchen Situation muss ein Stufenkonzept mit primärer Explantation des Spacers, Débridement und Biopsieentnahmen und, je nach mikrobiologischem Ergebnis und Antibiotikaregime, eine frühzeitige oder verzögerte Rekonstruktion des Azetabulums mit azetabulärem Aufbau und Implantation einer Hüftrevisionsprothese durchgeführt werden (Abb. 8).

\section{Fazit für die Praxis}

Für die Therapieentscheidung bei periazetabulären Frakturen bei Hüftprothesen müssen patienten-, fraktur- und prothesenspezifische Faktoren berücksichtigt werden.

Nicht oder wenig dislozierte Frakturen bei stabiler Hüftpfanne ohne azetabuläre Lysezonen können konservativ behandelt werden. Jedoch Frakturen mit Lysezonen und instabiler Pfanne und eigentliche akute Beckendiskontinuitäten müssen operativ angegangen werden. Häufig reicht die Osteosynthese des Hinterpfeilers und die Implantation einer Revisionspfanne, wobei die Implantation einer TM-Pfanne bessere Resultate aufweist als ein Revisionsring.

Durch die Etablierung von intrapelvinen Zugängen (modifizierter Stoppa-Zugang oder Pararectus-Zugang nach Keel) in der Azetabulumtraumatologie kann durch die Zuggurtung des vorderen Pfeilers ein stabiles „Down-grading“ des Knochendefekts mit guter Integration von verwendetem Allograft und/oder TM-Augment erreicht werden.

Langzeitresultate dieses Behandlungskonzepts bei akuten oder chronischen Beckendiskontinuitäten fehlen jedoch noch. Aus demografischen Gründen wird der Hüftchirurg in Zukunft häufiger mit komplexen Beckendiskontinuitäten konfrontiert sein. 

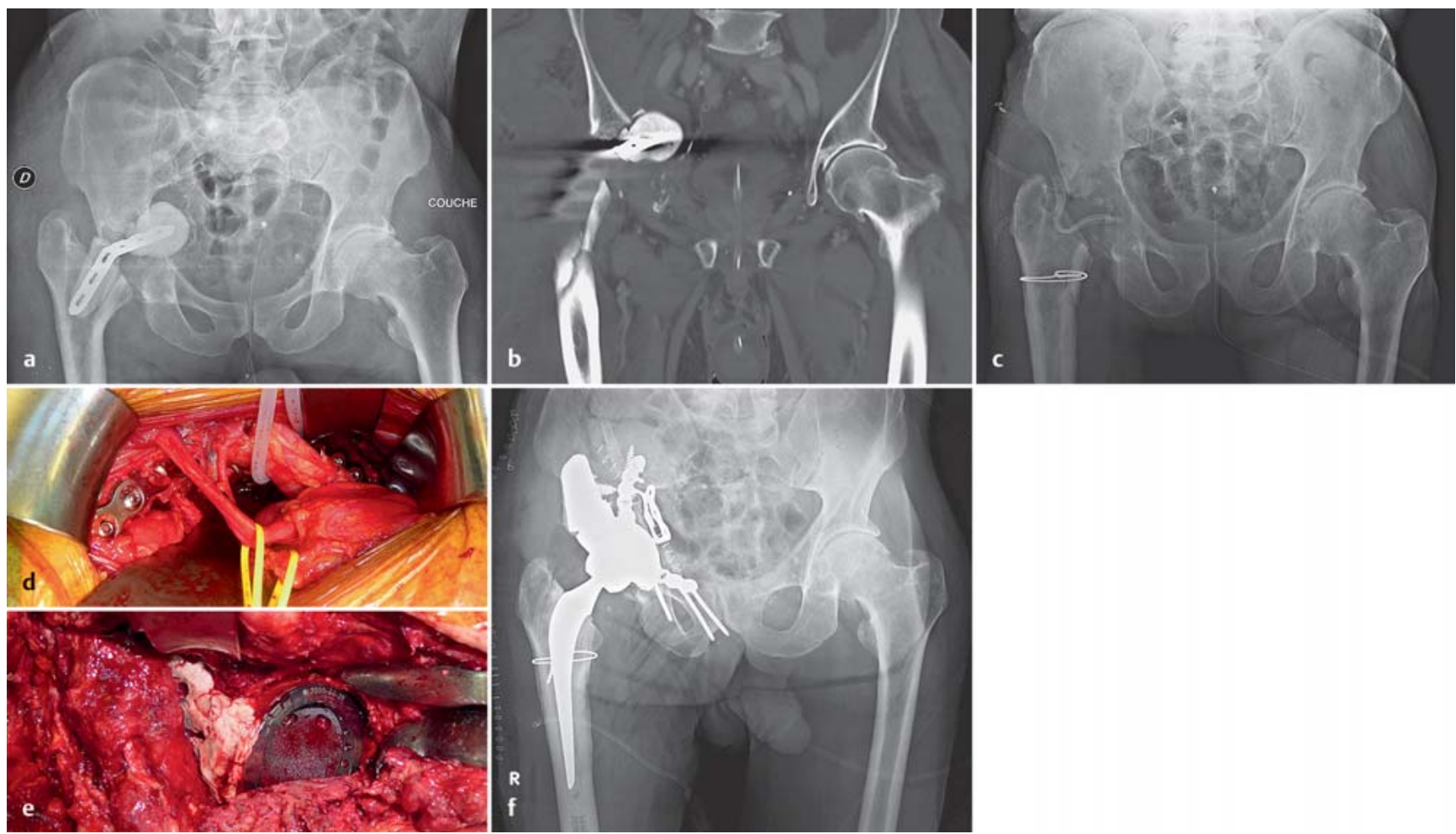

Abb. $\mathbf{8}$ a bis $\mathbf{f}$ Bei einem 78-jährigen Patienten entstanden nach einem Sturz bei liegendem Spacer nach Prothesenexplantation bei infizierter Hüfttotalprothese eine akute Beckendiskontinuität (Azetabulumquerfraktur) mit zentraler Luxation des Spacers und eine wenig dislozierte proximale Femurfraktur (a Beckenübersicht und b koronarer CT-Schnitt). Neben der Explantation des Spacers und Cerclage des proximalen Femurs wurde ein ausgedehntes Débridement initial durchgeführt (c Girdlestone-Situation). Bei infektfreier Situation wurde in einer Halbseitenlagerung über den Pararectus-Zugang der Vorderpfeiler mittels Plattenosteosynthese fixiert (d intraoperativer Situs mit angeschlungenen Ductus spermaticus, Vasa epigastrica und Vasa iliaca externa [von links nach rechts]) und über den transglutealen Zugang eine Hüftrevisionsprothese implantiert (e intraoperativer Situs mit TM-Pfanne zementiert an ein kraniodorsales Augment bei großem Hinterwand- und Domdefekt). $\mathbf{f}$ Die postoperative Beckenübersicht zeigt das kraniodorsale Augment mit der TM-Pfanne und die Spezialplatte auf dem vorderen Pfeiler mit kombinierter Abstützplatte auf der quadrilateralen Fläche.

\section{Literatur}

${ }^{1}$ Abolghasemian M, Tangsaraporn S, Drexler $M$ et al. The challenge of pelvic discontinuity. Cup-cage reconstruction does better than conventional cages in mid-term. Bone Joint J 2014; 96: 195-200

2 Bastian JD, Tannast M, Siebenrock KA et al. Mid-term results in relation to age and analysis of predictive factors after fixation of acetabular fractures using the modified Stoppa approach. Injury 2013; 44: 1793-1798

3 Beckmann NA, Weiss S, Klotz MCM et al. Loosening after acetabular revision: Comparison of trabecular metal and reinforcement rings. A systematic review. J Arthroplasty 2014; 29: $229-235$

${ }^{4}$ Chana-Rodriguez F, Villanueva-Martinez $M$, Crego-Vita $D$ et al. Stoppa approach, an alternative for total hip arthroplasty in an intrapelvic cup. J Arthroplasty 2013; 28: 988-993

${ }^{5}$ D'Antonio JA, Capello WN, Borden LS et al. Classification and management of acetabular abnormalitis in total hip arthroplasty. Clin Orthop Relat Res 1989; 243: 126-137

${ }^{6}$ Ferguson TA, Patel R, Bhandari $M$ et al. Fractures of the acetabulum in patients aged 60 years and older: an epidemiological and radiological study. J Bone Joint Surg Br 2010; 92: $250-257$

7 Jakobsen T, Kappel A, Hansen Fet al. The dislocating hip replacement - Revision with a dual mobility cup in 56 consecutive patients. Open Orthop J 2014; 8: 268-271
${ }^{8}$ Keel MJ, Ecker TM, Cullmann JL et al. The Pararectus approach for anterior intrapelvic management of acetabular fractures. An anatomical study and clinical evaluation. J Bone Joint Surg Br 2012; 94: 405-411

${ }^{9}$ Keel MJ, Ecker TM, Siebenrock KA et al. Rationales for the Bernese approaches in acetabular surgery. Eur J Trauma Emerg Surg 2012; 38; 489-498

10 Letournel E. Acetabulum fractures: classification and management. Clin Orthop 1980; 151: 81-106

11 Nieminen J, Pakarinen TK, Laitinen M. Orthopaedic reconstruction of complex pelvic bone defects. Evaluation of various treatment methods. Scand J Surg 2013; 102: 36-41

12 Paprosky WG, Perona PG, Lawrence JM. Acetabular defect classification and surgical reconstruction in revision arthroplasty: a 6year follow-up evaluation. J Arthroplasty 1994; 9: 33-44

13 Petrie J, Sassoon A, Haidukewych GJ. The revision hip. Pelvic discontinuity. Current solutions. Bone Joint J 2013; 95-B, Suppl. A: S109-S113

${ }^{14}$ Rogers BA, Whittingham-Jones PM, Mitchell PA et al. The reconstruction of periprosthetic pelvic discontinuity. J Arthroplasty 2012; 27: 1499-1506

15 Tam HH, Bhaludin B, Rahman F et al. SPECTCT in total hip arthroplasty. Clin Radiol 2014; 69: 82-95

\section{Prof. Dr. med. Marius Johann Baptist Keel, FACS}

Stellvertretender Chefarzt

Leiter für Traumatologie, Becken- und Wirbelsäulenchirurgie

Universitätsklinik für Orthopädische

Chirurgie und Traumatologie

Universitätsspital Bern, Inselspital

Freiburgstrasse 3

3010 Bern

Schweiz

marius.keel@insel.ch

\section{Dr. med. Johannes Dominik Bastian Oberarzt Hüftteam}

Universitätsklinik für Orthopädische Chirurgie und Traumatologie Universitätsspital Bern, Inselspital Freiburgstrasse 3

3010 Bern

Schweiz

johannes.bastian@insel.ch 